\title{
Corrigendum
}

Jindong Xie, Lieqian Chen, Dongqiang Huang, Weiwei Yue, Jingyu Chen*, Chunxiao Liu*

\section{Corrigendum to "A nitric oxide-releasing prodrug promotes apoptosis in human renal carcinoma cells: Involvement of reactive oxygen species"}

https://doi.org/10.1515/chem-2021-0556

received June 8, 2021; accepted June 10, 2021

Corrigendum to: Xie J, Chen L, Huang D, Yue W, Chen J, Liu C. A nitric oxide-releasing prodrug promotes apoptosis in human renal carcinoma cells: Involvement of reactive oxygen species. Open Chem. 2021;19(1):635-45. doi: 10.1515/chem-2020-0075.

After publishing the article, the authors noticed that the wrong Figure 4 was used in the manuscript.

* Corresponding author: Jingyu Chen, Department of Urology, The First Hospital of Huizhou, No. 20, Sanxin Road, Huizhou, 516000, Guangdong, China, e-mail: hzmiwai@163.com

* Corresponding author: Chunxiao Liu, Department of Urology, Zhujiang Hospital, Southern Medical University, No. 253, Industrial Road, Guangzhou, 510282, Guangdong, China,

e-mail: liuchx888@163.com

Jindong Xie: Department of Urology, Zhujiang Hospital, Southern Medical University, No. 253, Industrial Road, Guangzhou, 510282, Guangdong, China Lieqian Chen, Dongqiang Huang, Weiwei Yue: Department of Urology, The First Hospital of Huizhou, No. 20, Sanxin Road, Huizhou, 516000, Guangdong, China 
The correct version of Figure 4 is as follows:

(a)

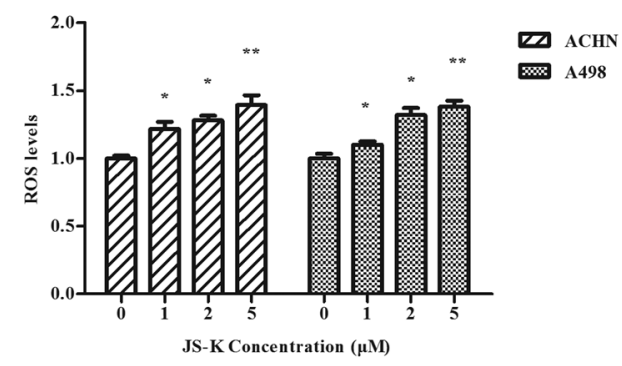

(c)

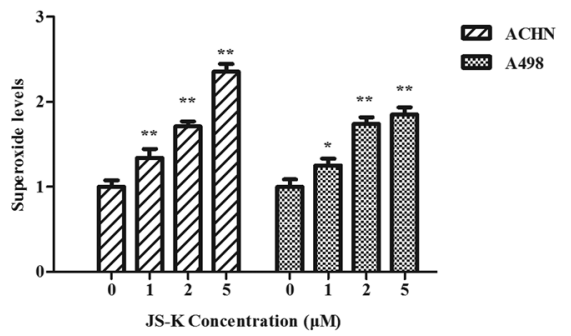

(e)

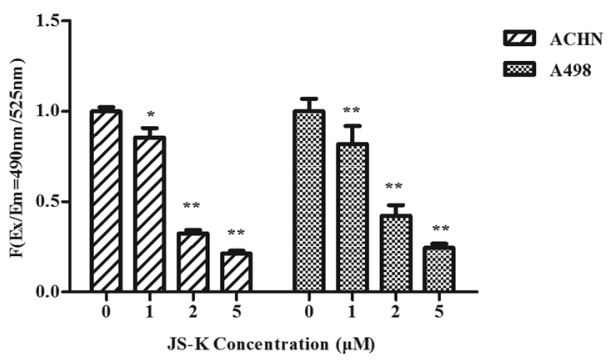

(b)

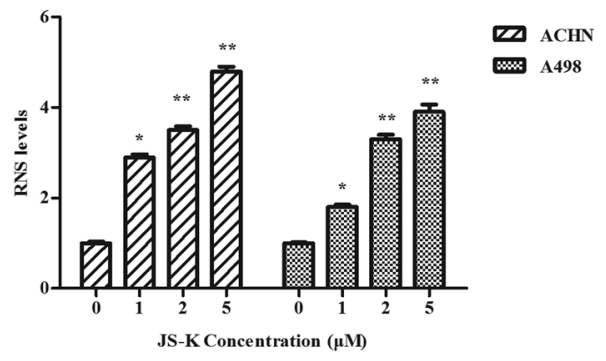

(d)

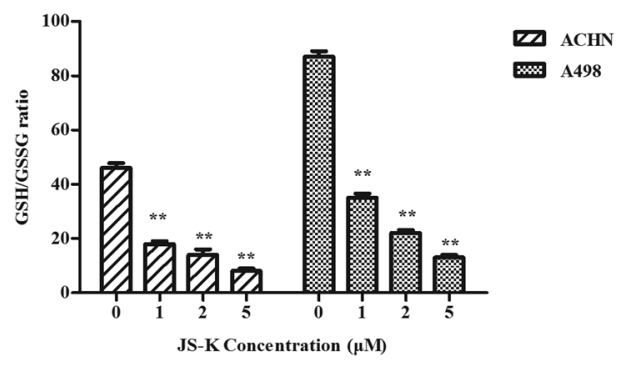

(f)

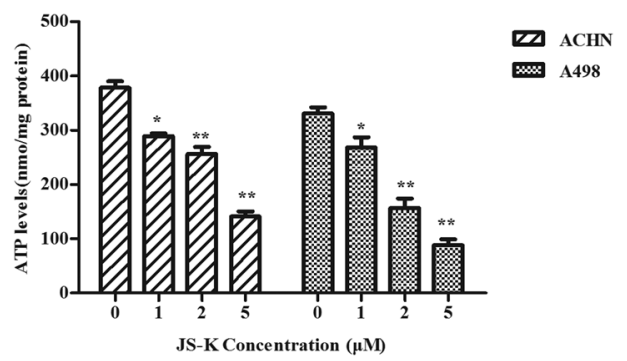

(g)

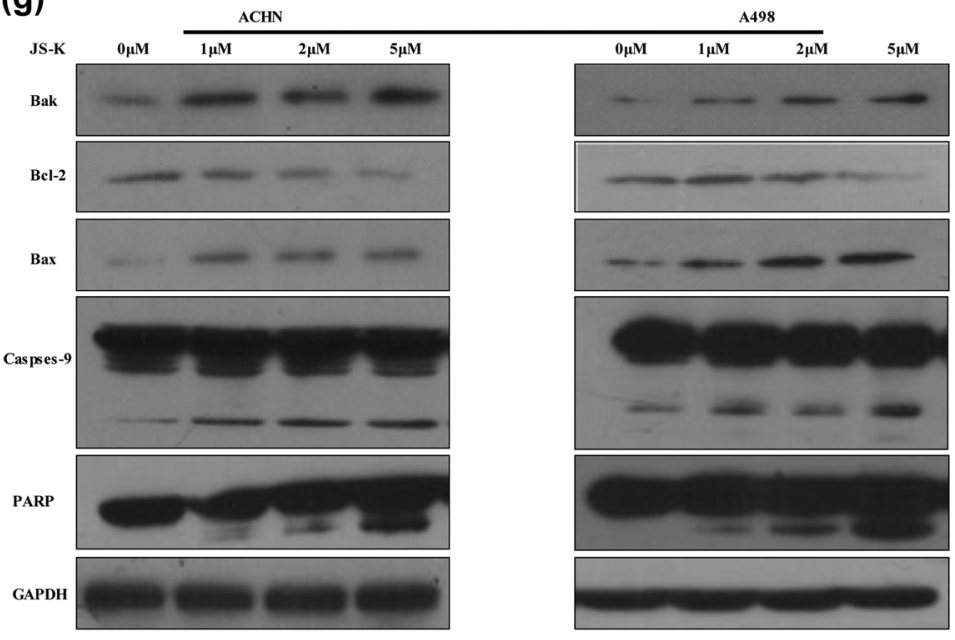

Figure 4: Effect of JS-K on ROS, RNS, mitochondrial membrane potential, GSH/GSSG ratio, ATP production, and apoptotic-related proteins in renal carcinoma cells. Treatment of JS-K renal carcinoma cells $(0,1,2$, and $5 \mu \mathrm{M})$ for $360 \mathrm{~min}$, and then detection of intracellular maximum ROS (a), RNS (b), superoxide (c), and GSH/GSSG ratio (d) levels. Upon 360 min of JS-K treatment, measurements were made for mitochondrial membrane potential (e) and ATP output (f). Western blot was used to identify concentrations of apoptotic-related proteins such as Bak, Bax, and PARP after cells were treated with JS-K $(0,1,2$, and $5 \mu \mathrm{M})$ for $24 \mathrm{~h}(\mathrm{~g})$. For at least three separate assays, findings are reported as mean \pm SD. 